ISSN: 2550 - 682X

DOI: $10.23857 / p c . v 4 i 1.864$

Recepción: 01 / 11 / 2018

Ciencias económicas y empresariales

Aceptación: 17 / 12 / 2018

Publicación: 20 / 01 / 2019

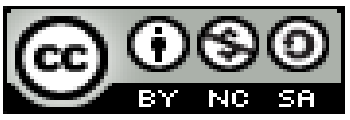

Articulo original

\title{
El valor nutricional de los desayunos que se ofertan en la gastronomía típica de la ciudad de Manta
}

\section{The nutritional value of the breakfasts that are offered in the typical cuisine of the city of Manta}

\section{O valor nutritivo dos cafés da manhã que são oferecidos na culinária típica da cidade de Manta}

\author{
Jessica M. Mendoza-Balcázar ${ }^{\mathrm{I}}$ \\ jessicamendozabalcazar@gmail.com \\ Mayra J. Ramírez-Bazurto II \\ ma_y_med10@hotmail.com
}

Correspondencia: jessicamendozabalcazar@gmail.com

\footnotetext{
I Magíster en Gerencia Educativa, Licenciada en la Especialización de Nutrición y Dietética, Docente de la Universidad Laica Eloy Alfaro de Manabí, Manta, Ecuador.

II Magíster en Gerencia de Salud para el Desarrollo Local, Magíster en Salud y Seguridad Ocupacional Mención en Prevención de Riesgos Laborales, Ingeniera Comercial, Médico Cirujano, Docente de la Universidad Laica Eloy Alfaro de Manabí, Manta, Ecuador.
} 


\section{Resumen}

La gastronomía en Manta por su ubicación geográfica de perfil costanero y áreas montañosas, como el bosque de Pacoche, por su clima subtropical a templado marítimo proporcionan diversidad de alimentos tanto de mar como de tierra; alimentos frescos con sus propios códigos tales como el color olor y sabor característicos de cada zona donde se produce, que se dan en base a su geografía, tipo de cocción e instrumentos culinarios artesanales e industriales, siendo la gastronomía una fuerte carta de presentación, causando gran aceptación en todos sus habitantes, satisfaciendo a los paladares más exigentes de locales como de turistas. Se utilizaron métodos de investigación teóricos y empíricos, dados fundamentalmente en estudios de cuáles son los alimentos de mayor consumo, con su proceso autóctono de preparación y cocción, lo que facilitó el análisis nutricional de los mismos; además se utilizó métodos empíricos como la técnica de encuestas, con un instrumento de diez preguntas cerradas. Se analizó los documentos de política pública oficiales empleados en Ecuador, como La Ley Orgánica del Régimen de la Soberanía Alimentaria vigente del Ecuador, y la tabla de composición química de los alimentos. Con la información se procedió a identificar los desayunos de mayor consumo en la gastronomía típica de Manta y las bebidas que lo acompañan en conjunto con los factores que determinan su elección.

Palabras clave: Gastronomía; valor nutricional; comida típica; alimentos; variedad.

\section{Abstract}

The gastronomy in Manta due to its geographical location with a coastal profile and mountainous areas, such as the Pacoche forest, due to its subtropical to temperate maritime climate, provides diversity of food both from sea and land; fresh food with its own codes such as the smell and flavor color characteristic of each area where it is produced, which are given on the basis of its geography, type of cooking and industrial and artisan culinary instruments, gastronomy being a strong letter of introduction, causing great acceptance in all its inhabitants, satisfying the most demanding palates of locals and tourists. Theoretical and empirical research methods were used, given mainly in studies, of which are the most consumed foods, with their native process of preparation and cooking, which facilitated the nutritional analysis of the same; In addition, empirical methods such as the survey technique were used, with an instrument of ten closed questions. The official public policy documents used in Ecuador were analyzed, such as the 
Organic Law of the Current Food Sovereignty Regime of Ecuador, and the table of chemical composition of food. With the information, we proceeded to identify the breakfasts with the highest consumption in the typical cuisine of Manta and the drinks that accompany it together with the factors that determine their choice.

Keywords: Gastronomy; Nutritional value; typical food; foods; variety.

\section{Resumo}

Gastronomia em Manta para a sua localização geográfica e áreas de perfis costeiras montanhosas como a floresta Pacoche, pelo seu clima subtropical temperado marítimo fornecer diversidade alimentar tanto mar e terra; alimentos frescos com seus próprios códigos, tais como odor cor e sabor de cada região onde é produzido, que são dadas com base na geografia, tipo de culinária e artesanato e instrumentos culinários industriais, com a gastronomia uma carta de apresentação forte, causando grande aceitação em todos os seus habitantes, satisfazendo os paladares mais exigentes dos habitantes locais e turistas. métodos de investigação teórica e empírica, dada principalmente em estudos do que a maioria dos alimentos consumidos com seu processo de preparação e cozinhar nativa utilizada, o que facilitou a análise nutricional dos mesmos; Além disso, foram utilizados métodos empíricos, como a técnica de pesquisa, com um instrumento de dez questões fechadas. Foram analisados os documentos oficiais de política pública usados no Equador, como a Lei Orgânica do atual Regime de Soberania Alimentar do Equador e a tabela de composição química dos alimentos. Com a informação procedeu-se a identificar os cafés da manhã com maior consumo na cozinha típica de Manta e as bebidas que a acompanham juntamente com os fatores que determinam sua escolha.

Palavras chave: Gastronomia; valor nutricional; comida típica; comida; variedade.

\section{Introducción}

El Cantón Manta de la provincia de Manabí, es conocido como puerto pesquero por su gran aporte a la economía en todos sus ámbitos, por sus diversas industrias alimenticias que empujan al desarrollo del cantón. Anualmente se reciben cerca de 600.000 turistas nacionales; su puerto de aguas profundas permite la llegada de cruceros de diversas nacionalidades, en los que arriban aproxidamente unos 150.000 turistas extranjeros. Su diversidad geográfica se destaca en atractivos naturales, lo que aumenta la economía y atrae a los turistas incrementando los 
huéspedes en la industria hotelera, la gastronomía se empodera como carta de presentación de la cultura, su alimentación con ingredientes propios de la zona, por lo que se tomó la decisión de realizar un estudio del valor nutricional de los desayunos que se ofertan como alimentos típicos gastronómicos para realzar y mejorar sus preparaciones, no excluirlos, ya que la comida Manabita es considerada patrimonio inmaterial por el ministerio de Cultura Del Ecuador, por los ingredientes propios de cada zona y su uso en diversas preparaciones con aportes nutricionales energéticos, equilibrados y balanceados.

\section{Metodología}

La presente investigación tiene el siguiente objetivo general: Determinar cuáles son los desayunos mayormente consumidos de la gastronomía típica del cantón Manta para incidir en diversos tipos de menús equilibrados y balanceados nutricionalmente.

Para lograr el objetivo de la investigación se establecen los siguientes objetivos específicos:

- Evaluar el contenido nutricional de los desayunos mayormente consumidos

- Realizar propuestas de mejoras al contenido nutricional de los desayunos típicos de Manta

\section{Metodología aplicada}

Llegando a este punto de la investigación, donde se explica en detalle los resultados obtenidos a partir de los objetivos, se organizará el estudio para dar respuesta a lo planteado.

\section{Técnicas utilizadas}

Las técnicas utilizadas en la investigación son observación, fichas, encuestas, La observación se hará en porciones y raciones de los alimentos expendidos y sus menús en los lugares de estudio, mediante un trabajo de campo.

Para la realización de las fichas, se elaboró un banco de preguntas de los aspectos de mayor relevancia que brinde información oportuna de la lista de alimentos de la gastronomía típica de mayor expendio; para la aplicación de las encuestas se realizará un plan de preguntas, las cuales alojen datos exactos de los alimentos de mayor consumo, los factores que determinan su consumo y su bebida.

\section{Resultados}


El diagnóstico de los productos inventariados, se explicará a través de una tabla, tomando en cuenta el número de consumidores.

Gráfico 1. Lista de alimentos gastronómicos típicos de mayor consumo.

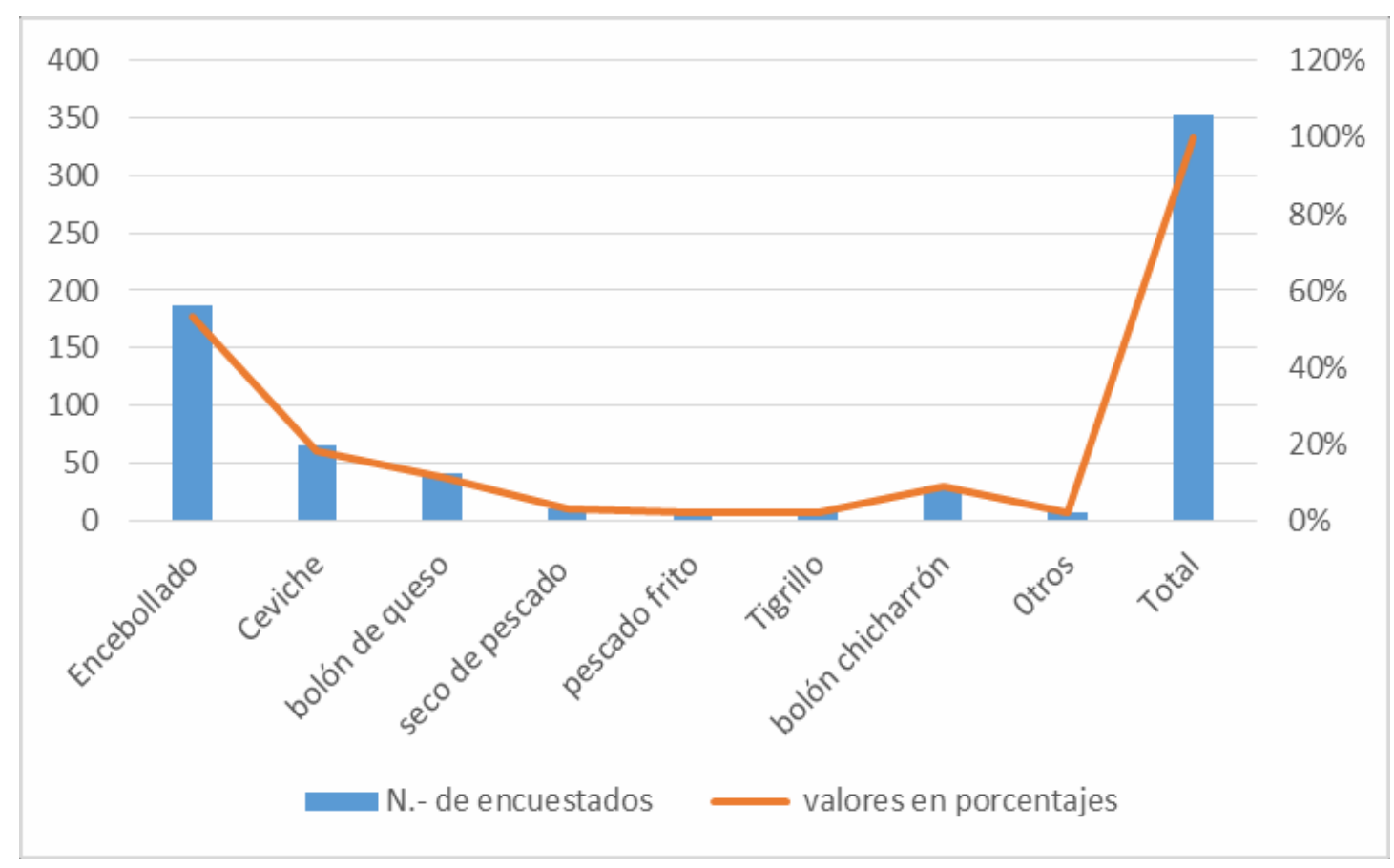

Fuente: consumidores de alimentos de parroquias urbanas de Manta

Los establecimientos que se dedican a la venta de comida típica gastronómica, están conscientes de la gran aceptación que tienen los platos que contienen mariscos, por su variedad, precio y frescura, uno de estos es el encebollado, que es altamente consumido y expendido en muchos locales, ya que los ingredientes son propios de la localidad, como el pescado, cebolla perla, cilantro, yuca, ají peruano acompañado de chifles.

Existen diversos tipos de pescados marítimos, siendo los más utilizados en la lista de alimentos de mayor consumo: albacora, bonito, dorado con aportaciones altas de ácidos grasos poliinsaturados, y proteínas de alta calidad. 
Gráfico 2. Las bebidas de mayor consumo en el desayuno

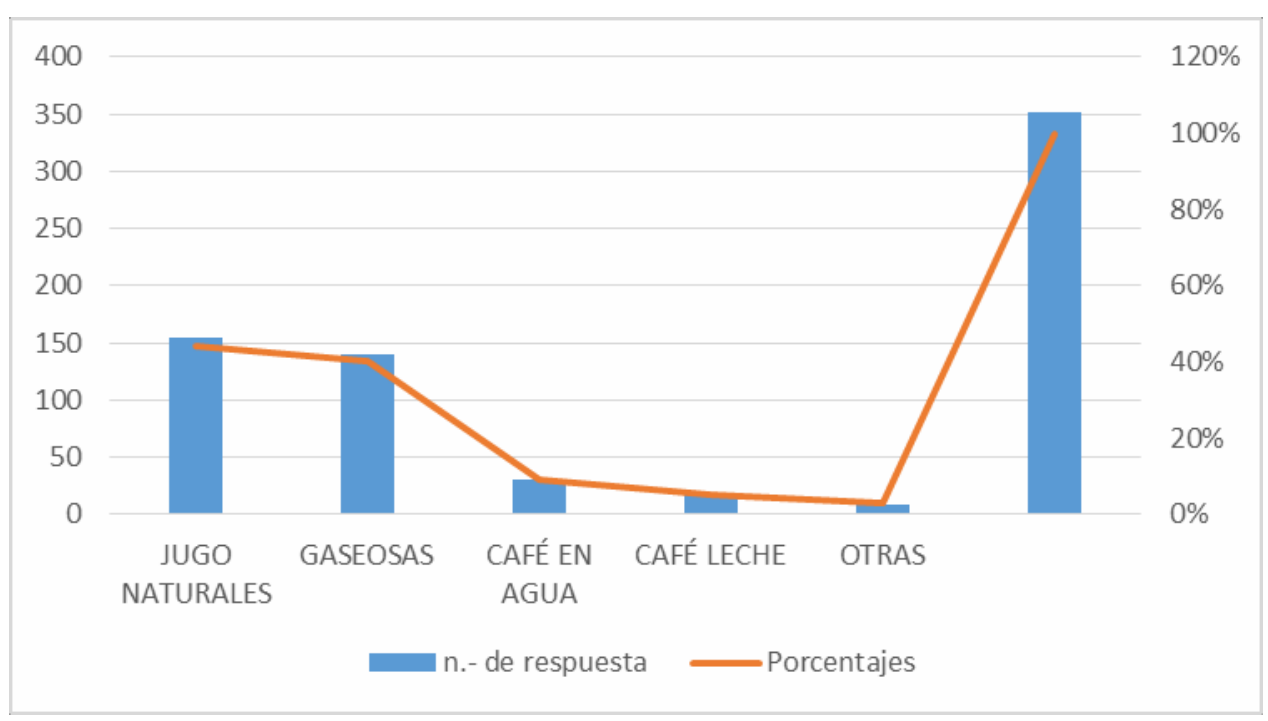

Fuente: consumidores de alimentos de parroquias urbanas de Manta

Las bebidas que tienen mayor consumo son los jugos naturales, con azúcar adicional, lo que aumenta las calorías en la ingesta de los desayunos, seguido por las gaseosas que por su alto de calorías-azúcar, se transforma en grasa en el organismo

Gráfico 3. Tipos de aderezos adicionados al desayuno.

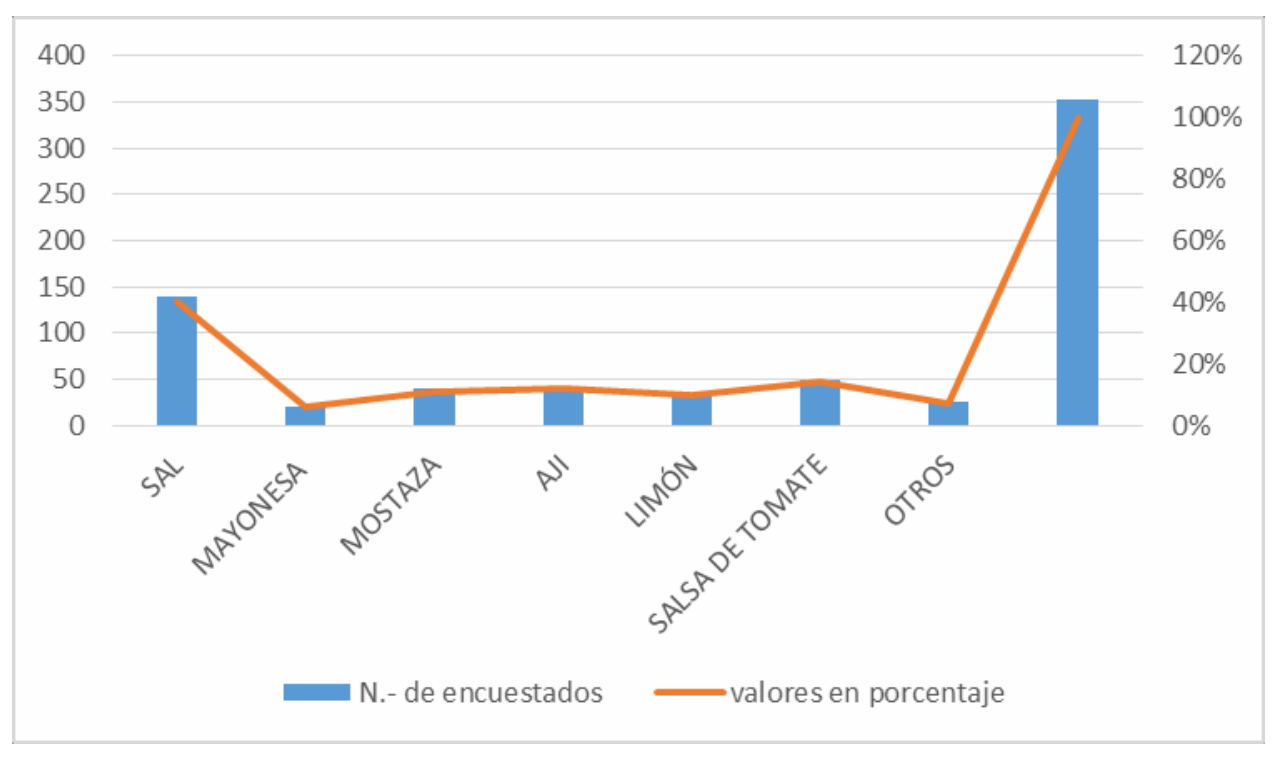

Fuente: Consumidores de alimentos de parroquias urbanas de Manta 
El aderezo de mayor consumo es la sal de mesa, seguido de la salsa de tomate y mostaza, las que tienen un alto contenido en sodio; su consumo en cantidades normales muestran beneficios como relajación muscular y balance hídrico, mientras que su ingesta en exceso, provoca una necesidad de aumentar el consumo de agua, retención de líquido, niveles de presión arterial alta, aumentando el riesgo de morbilidad; en sí, los mariscos aportan gran cantidad de yodo, que es el encargado de nivelar los niveles normales de la hormona tiroides. Los productos procesados tienen un alto contenido de preservantes. Estos hábitos forman la cultura alimentaria, tanto en su aspecto culinario como gastronómico y sodio por lo que su uso frecuente causa un desbalance en la salud. Dentro de las políticas de estado de seguridad alimentaria, la sal de mesa contiene flúor y yodo, minerales esenciales que ayudan al equilibrio homeostático del organismo.

Gráfico 4. Horarios de Desayuno

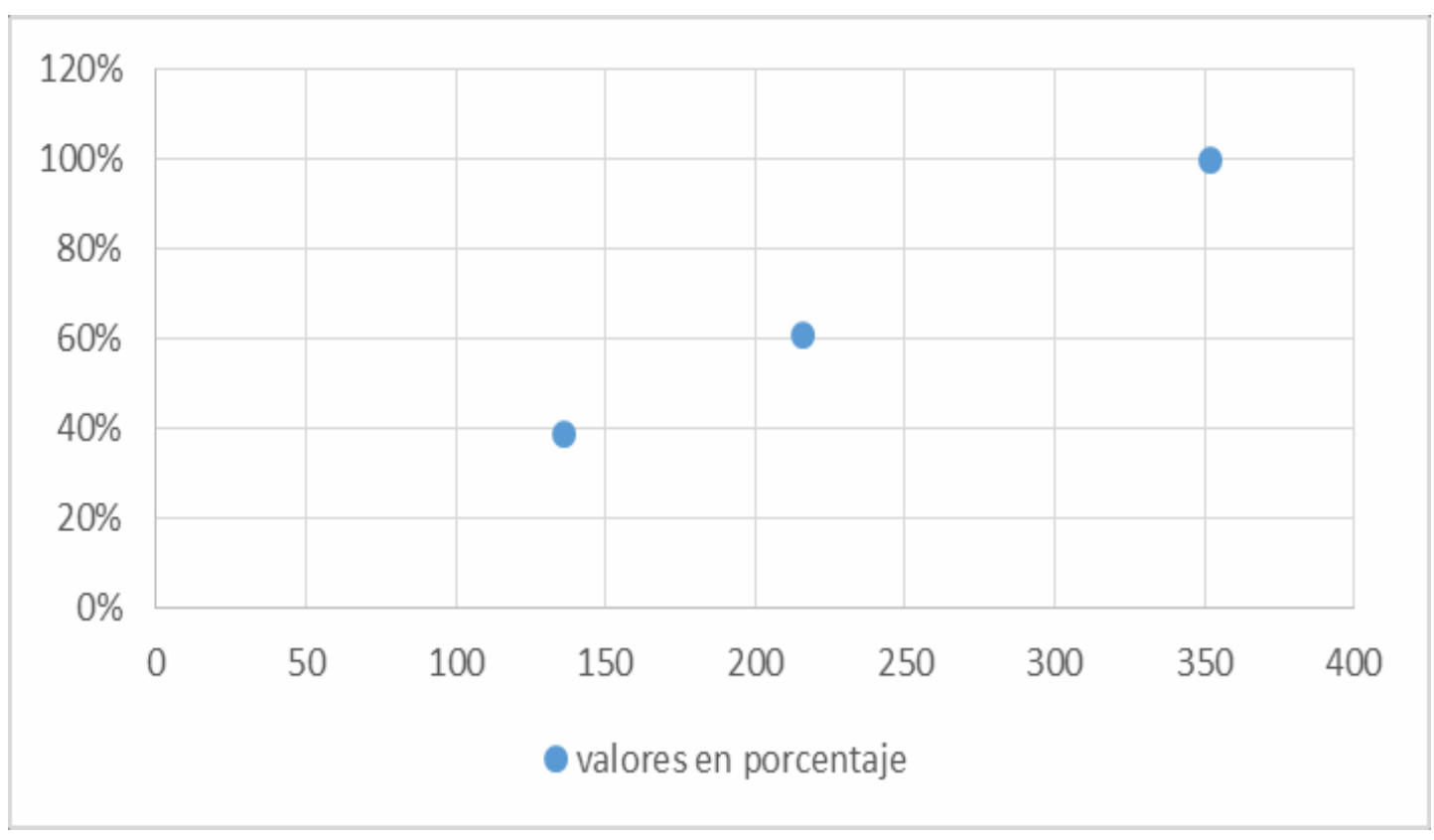

Fuente: consumidores de alimentos de parroquias urbanas de Manta

El tener horarios fijos en las comidas principales es de mucha importancia ya que nivela el metabolismo, el desayuno es de vital importancia puesto que en él se genera la energía necesaria para realizar las actividades cotidianas, y evita la ansiedad de comer reiteradas veces entre comidas. 


\section{Discusión}

\section{Importancia del Problema}

La importancia de la gastronomía típica en Manta crece día a día, por su gran incremento turístico, por sus ingredientes autóctonos, lo cual se deben de mantener; el problema radica en los aderezos que se incluyen en la preparación de estos alimentos, como la sal, ají, salsa de tomate, mostaza; sus usos frecuentes en cantidades mayores ha provocado en la población un aumento en los niveles de presión arterial, según datos proporcionados por el Ministerio de Salud Pública en la encuesta Nacional de Salud y Nutrición, casi el 50\% de los ecuatorianos de entre 10 y 59 años son pre-hipertensos y cerca del $20 \%$ son hipertensos llegando a un número de 717.529 personas.

\section{Valor nutricional del encebollado}

\section{Porción: 1}

Medida casera: plato sopero grande

- $\quad$ Yuca 25 gr.

- $\quad$ Pescado 100 gr.

- Cebolla colorada 20 gr.

- $\quad$ Ají peruano 5 gr.

- Cilantro 5 gr.

- $\quad$ Chifles 50 gr.

Tabla 1. Valor nutricional de yuca blanca en 25 gr.

\begin{tabular}{lc}
\hline Nutrientes & Contenido \\
\hline valor calorías & 36,50 \\
HCO & 6,72 \\
Proteina & 0,80 \\
Grasa & 0,10 \\
Vitamina C & 12,00 \\
vitamina B6 & 0,015 \\
Potasio & 191,00 \\
Magnesio & 16,15 \\
\hline
\end{tabular}


La yuca se cultiva principalmente en América, Asia y África, donde la utilizan en su gastronomía tradicional para preparar multitud de recetas culinarias. De una de las variedades de yuca dulce se obtiene la tapioca.

Nutricionalmente, la yuca es un tubérculo, alimento beneficioso para introducir en la dieta, ya que, cuenta con propiedades nutricionales similares a la papa, o camote, siendo muy rica en hidratos de carbono complejos, es decir, que aporta mucha energía. Se cosecha de manera abundante en el Oriente y Manabí; los cantones con mayor producción en Manabí son el Carmen y Canuto, de Chobe, por la complejidad del suelo.

Tabla 2. Valor nutricional cebolla paiteña en 20 gr.

\begin{tabular}{lrlr}
\hline Nutrientes & Contenido & Nutrientes & Contenido \\
\hline Agua & 17,8 & Fosforo & 6,6 \\
Calorías & 7,6 & Calcio & 3 \\
Grasas & 0,04 & Magnesio & 2 \\
Carbohidratos & 2,15 & Hierro & 0,033 \\
Proteínas & 0,29 & Vitamina C & 12,68 \\
Fibras & 0,35 & Vitamina E & 0,052 \\
Potasio & 31,4 & Vitamina B6 & 0,0232 \\
Azufre & 14 & & \\
\hline
\end{tabular}

Tabla 3. Valor nutricional del limón 10 gr.

\begin{tabular}{lr}
\hline Nutrientes & Contenido \\
\hline Calorías & 2,3 \\
HCO & 0,9 \\
Folatos & 1,8 \\
Potasio mg. & 14,9 \\
Magnesio mg. & 1,8 \\
Ácido ascórbico & 4,8 \\
\hline
\end{tabular}

El limón, fruta de ambiente templado le adiciona un sabor ácido a las preparaciones, protege el sistema inmunitario, rico en folatos y vitamina C. Se produce de manera abundante en Manabí, en mayor parte en el sector Ayacucho del cantón Santa Ana. 
Tabla 4. Valor nutricional del plátano en chifles 50 gr.

\begin{tabular}{lrlr}
\hline Nutrientes & Contenido & Nutrientes & Contenido \\
\hline Agua & 32,65 & sodio & 270 \\
Proteína & 1 & Potasio & 243 \\
Grasa total & 16 & Calcio & 1,5 \\
Grasa saturada & 9 & Magnesio & 18,5 \\
$\begin{array}{l}\text { Ácidos grasos } \\
\text { monoinsaturados }\end{array}$ & 7 & Hierro & 0,3 \\
Ácidos grasos & & & \\
poilinsaturados & 1 & Fosforo & 17 \\
Carbohidratos totales & 25 & Zinc & 0,7 \\
Vitamina B2 & 0,025 & calorías & 260 \\
Vitamina B6 & 0,15 & & \\
\hline
\end{tabular}

Tabla 5. Valor nutricional del ají peruano 5 gr.

\begin{tabular}{lr}
\hline Nutriente & Contenido \\
\hline Calorías & 5,2 \\
Proteína & 0,13 \\
Grasa Total & 0,17 \\
Carbohidratos & 0,32 \\
Fibra & 0,3 \\
Calcio & 3,7 \\
Hierro & 0,35 \\
Vitamina C & 1,62 \\
\hline
\end{tabular}

Tabla 6. Valor nutricional de la albacora en $100 \mathrm{gr}$.

\begin{tabular}{lrlr}
\hline Nutriente & Contenido & Nutriente & Contenido \\
\hline Calorías & 200 & Zinc & 1,1 \\
Proteína & 23 & Fósforo & 200 \\
Grasas & 12 & Sodio & 43 \\
Agua & 65 & Niacina & 17,8 \\
Calcio & 38 & Vitamina D & 25 \\
Hierro & 1,3 & Vitamina A & 60 \\
Yodo & 8 & Potasio & 40 \\
Magnesio & 28 & & \\
\hline
\end{tabular}

Son alimentos semejantes a la carne, pero con un contenido en proteína ligeramente inferior (15\%) y con una cantidad de tejido conectivo mucho menor, lo que hace que sean más fáciles de digerir. Son buena fuente de vitamina $\mathrm{D}$ y yodo, y son ricos en ácidos grasos poliinsaturados de 
la serie n-3, sobre todo los pescados azules como el arenque, atún, bonito, salmón y sardina. Estos ácidos grasos son precursores de eicosanoides (tromboxanos y prostaciclinas) con efectos cardiovasculares beneficiosos (Ros y Martínez, 2005).

Los mariscos se caracterizan por tener un bajo contenido en grasa saturada y alto en proteína; además son una buena fuente de vitaminas del grupo B como la tiamina, y de minerales como hierro, yodo y zinc.

\section{Conclusiones}

- $\quad$ El alimento de mayor consumo en los desayunos del cantón Manta, es el encebollado.

- $\quad$ En general, la carne de pescado es la más consumida, rica en grasos poliinsaturados.

- La ubicación geográfica de Manta da la facilidad estratégica en adquisición de ingredientes organolépticos, los cuales realzan su gastronomía.

Tanto mujeres como hombres expenden alimentos típicos.

El valor nutricional en calorías del encebollado es de 439 calorías.

\section{Referencias Bibliográficas}

Tratado de nutrición. Ediciones Díaz de Santos, 1999. ProQuest Ebook Central. Disponible en: http://ebookcentral.proquest.com/lib/uleamecsp/detail.action?docID=3175376

Mañas, Almendros, Mariano, et al., 2010. Principios generales de nutrición. Ediciones Díaz de Santos. ProQuest Ebook Central. Disponible http://ebookcentral.proquest.com/lib/uleamecsp/detail.action?docID=3228516.

El Comercio, 2016. El Patrimonio Manabita está en su gastronomía,

El Comercio, 2015. El ceviche, apanado y encebollado de pescado se imponen en las huecas de Manta.

El Telégrafo, 2016. Huecas, el encebollado, ceviche de pichagua, más buscados en Manta.

El Telégrafo, 2018. Manta es todo. 